\title{
Numerical Investigation on the Effects of a Precursor Wetting Film on Immiscible Displacement along a Channel
}

\author{
Kai $\mathrm{Bao}^{1}$, Amgad Salama, ${ }^{2, *}$, and Shuyu Sun ${ }^{2}$ \\ ${ }^{1}$ SINTEF, Department of Applied Mathematics, Forskningsveien 1, 0373, Oslo, Norway \\ ${ }^{2}$ King Abdullah University of Science and Technology (KAUST), Thuwal 23955-6900, KSA \\ (*email: amgad.salama@kaust.edu.sa, Phone: +966 128080 324)
}

\begin{abstract}
A set of numerical experiments has been conducted to study the effect of a precursor fluid layer on the motion of two phase system in a channel. This system is characterized by coupled CahnHillard and Navier-Stokes system together with slip boundary conditions. The solution of the governing equation involves first the solution of Cahn-Hillard equation with semi-implicit and Mixed finite element discritization with a convex splitting scheme. The Navier-Stokes equations are then solved with a P2-P0 mixed finite element method. Three cases have been investigated; in the first the effect of different wettability scenarios with no precursor layer has been investigated. In the second scenario, the effect of the precursor layer for different wettability conditions is investigated. In the third case, the effect of the thickness of the precursor layer is investigated. It is found that, wettability conditions have considerable effect on the flow of the considered two-phase system. Furthermore the existence of the precursor layer has additional influence on the breakthrough of the phases.
\end{abstract}

Keywords: Two-phase flow, precursor wetting layer, moving contact line, coupled Cahn-Hillard and Navier-Stokes system.

\section{Introduction}

It has been a common practice to water the garden before an expected storm to facilitate the drainage of water into the subsurface. It has also been noticed, following rain droplet over the surface of the front wind shield of a car, that when the glass is dry water drops stick to the surface until reaching some critical size when they break downwards because of gravity. Next water drop, even less than this critical size, will move fast following the trajectory of the 
previous drop. This and many others suggest that introducing a precursor layer of fluid into the surface largely facilitates the movement of fluid than it is if the surface is dry. In porous media applications this phenomenon is particularly important in initiating a good draining capacity of the soil. In fact very dry soil, theoretically, will not conduct fluids at all unless very high pressure is applied. It is only because of the residual fluids, which stick with the soil grains, that flow is initially facilitated. Experiments show that the soil always retains a small amount of fluid no matter how much suction one may impose. This is called residual saturation, which is, in part, due to fluid films coating the grains of the soil. In fact many of the observed fingering phenomena during the percolation of water into relatively dry soil are due, in part, to variations of relative permeability or, more accurately, to residual saturation inhomogeneity. In multiphase subsurface flow, two driving forces usually exist; one is due to gradient in static pressure and the other is due to gradient in capillary pressure in addition to other body forces (e.g., gravity). These two gradient forces have been suggested to be functions of phase saturations. In particular, for the force which is due to gradient in static pressure, the concept of relative permeability has been introduced to highlight the extra resistance to the flow in multiphase system. This resistance is the least when the pore space is fully saturated with one phase. On the other hand, the force which is due to gradient in capillary pressure is more complicated by the fact that capillary pressure is a function of contact angle, pore characteristic length scale and surface tension. That is gradient in capillary pressure could result as a consequence of gradients of either or all of these previously mentioned factors. In particular, spatial variations of wettability or surface tension changes due to temperature gradients are all factors affecting gradients of capillary pressure. It is expected, therefore, that fluid movement in pore space will be different if the surface of the pore space is; for example, coated with a precursor layer of fluid versus if the surface is dry. In this work, we conduct a set of simple pore scale numerical experiments to highlight the effects of the existence of a precursor layer of a fluid on the surface of channel on the flow characteristics of two-phase system.

Although variables at the porous media scale represent averaged quantities of pore scale quantities within a representative volume [e.g., 1-3], at the pore scale, such averaging is not required and primitive investigation could highlight more insight into multiphase flows at porous media scale. We highlight two main issues; the first is related to the effect of the thickness of the precursor layer and the second on the effects of different wettability conditions of the precursor 
fluid layer on flow characteristics. In this simulation the so called moving contact line problem becomes an issue. The contact line is the line where three phases meet (namely; the solid phase and two fluid phases). At the contact line the regular no-slip condition does not work and researchers suggested slippage through different models [4-13]. In this work which considers pressure-driven flows, the interface configuration is expected to be complex. The coupled Navier-Stokes, Cahn-Hillard framework [14-20], in which the interface is resolved, is considered as will be detailed later.

\section{On the moving contact line problem}

Since the moving contact line (MCL) plays important role in this research, a highlight on the essence of this subject is indeed beneficial. The moving contact line problem has puzzled researchers for quite a while for its apparent contradiction with the widely accepted no slip condition. The assumption of no slip condition at the interface between fluid and solid substrate is based on observations at macroscopic scales. However, at small scale, slippage has been observed and recent molecular dynamic simulations confirm such phenomena [21-25]. In fact Navier considered fluid slippage at the solid interface by introducing a slip length $\mathscr{L}$, which is the length along the normal to the interface where the extrapolated tangential velocity vanishes. Navier suggested that the slip is dependent on the shear rate at the solid interface. Such slip length is usually very small [21-25] and the slippage velocity scales with the macroscopic fluid velocity as $\mathscr{L} / L$ where $L$ is some macroscopic length scale. This explains why in macroscopic systems such slippage may be ignored. The no slip condition has also been challenged in relation to the problem of moving contact line (the line where a solid phase intersects with two immiscible fluids) even at the macroscopic scale. Molecular dynamics as that conducted by Qian et al. [26] on Couette geometry suggest that fluids molecules at the solid surface indeed move and in the vicinity of the contact line (CL) there is clear slip whereas far from the CL slippage is negligibly small as depicted schematically in Fig.1. To account for flow slippage in the vicinity of the CL, a number of models have been proposed, a good list of which can be found in [25]. Of these models, the generalized Navier boundary condition (GNBC) has shown to account comparably with the MD simulation. Therefore, in this work we consider the GNBC together with the coupled Cahn-Hillard, Navier-Stokes system. 


\section{Governing equations and Numerical Scheme}

To solve the two-phase problem with different wettability, a diffuse-interface formulation with the GNBC is used to handle the interface evolution together with the movement of the contact lines in an energy based framework. Different aspects of the equations and related numerical algorithms have been presented previously [14, 17 and 18]. The method used in the work follows closely our recent work on the finite element solution of the coupled Navier-Stokes and CahnHilliard equations [18]. For the sake of completion, however, we briefly introduce the governing equations and the employed numerical scheme.

Following [18], the following coupled Navier-Stokes and Cahn-Hilliard equations along with the GNBC are used in this study.

$$
\begin{gathered}
R\left(\frac{\partial \mathbf{u}}{\partial t}+\mathbf{u} \cdot \nabla \mathbf{u}\right)=-\nabla p+\Delta \mathbf{u}+\lambda \mu \nabla \phi \quad \text { in } \Omega \\
\nabla \cdot \mathbf{u}=0 \quad \text { in } \Omega \\
\frac{\partial \phi}{\partial t}+\mathbf{u} \cdot \nabla \phi=L_{d} \Delta \mu \quad \text { in } \Omega \\
\mu=-\varepsilon \Delta \phi-\frac{1}{\varepsilon}\left(\phi-\phi^{3}\right) \quad \text { in } \Omega
\end{gathered}
$$

As mentioned earlier, at the contact line, which represents the intersection of three interfaces, the fluids slip according to the generalized Navier boundary condition [14], such that

$$
\begin{gathered}
\beta u_{\tau}^{\text {slip }}=-\eta\left(\partial_{n} u_{\tau}+\partial_{\tau} u_{n}\right)+L(\phi) \partial_{\tau} \phi \quad \text { on } \Gamma_{\text {slip }} \\
\frac{\partial \phi}{\partial t}+u_{\tau} \partial_{\tau} \phi=-\Gamma(L(\phi)) \quad \text { on } \Gamma_{\text {slip }}
\end{gathered}
$$

where, $p$ is the pressure, $\mathbf{u}=\left(u_{x}, u_{y}\right)$ is the velocity, $\rho$ is the mass density, $\eta$ is the dynamic viscosity, $M$ is the phenomenological mobility coefficient, $\phi$ is the phase field value, $\mu$ is the chemical potential and $\mu=-K \Delta \phi-r \phi+u \phi^{3}, \mu \nabla \phi$ is the capillary force, with $K, r$ and $u$ are parameters related to the interface thickness $\xi=\sqrt{K / r}$, the interfacial tension $\gamma=2 \sqrt{2} r^{2} \xi / 3 u$, and the two homogeneous equilibrium phases $\phi_{ \pm}= \pm \sqrt{r / u}(= \pm 1$ in the paper). In the paper, the 
effects of the wettability are investigated, and constant mass density $\rho$ and dynamic viscosity $\eta$ are assumed.

$L(\phi)=K \partial_{n} \phi+\partial \gamma_{\omega f}(\phi) / \partial \phi, \quad \gamma_{\omega f}(\phi)=-\frac{1}{2} \gamma \cos \theta_{s} \sin \left(\frac{\pi}{2} \phi\right), \quad \theta_{s}$ is the static contact angle, $u_{n}=\mathbf{u} \cdot \mathbf{n}$ and $u_{\tau}=\mathbf{u} \cdot \boldsymbol{\tau}$ are the normal and tangent velocity on the wall boundary respectively, where $\mathbf{n}$ and $\boldsymbol{\tau}$ are the unit normal and tangent vectors at the slip boundary, respectively, $\beta$ is the slip coefficient.

The equations for the coupled system, are nondimensionlized by defining the reference length $L_{0}$, the reference velocity $V$ and the reference time, $L_{0} / V$, to yield the following system

$$
\begin{gathered}
R\left(\frac{\partial \mathbf{u}}{\partial t}+\mathbf{u} \cdot \nabla \mathbf{u}\right)=-\nabla p+\Delta \mathbf{u}+\lambda \mu \nabla \phi \quad \text { in } \Omega \\
\nabla \cdot \mathbf{u}=0 \quad \text { in } \Omega \\
\frac{\partial \phi}{\partial t}+\mathbf{u} \cdot \nabla \phi=L_{d} \Delta \mu \quad \text { in } \Omega \\
\mu=-\varepsilon \Delta \phi-\frac{1}{\varepsilon}\left(\phi-\phi^{3}\right) \quad \text { in } \Omega
\end{gathered}
$$

In this system, $\varepsilon$ is the ratio between interface thickness $\xi$ and reference length $L_{0}$. The boundary conditions along the slip boundary can, therefore, be reformulated as follows.

$$
\begin{gathered}
\frac{u_{\tau}^{s l i p}}{L_{s}}=-\left(\partial_{n} u_{\tau}+\partial_{\tau} u_{n}\right)+\lambda L(\phi) \partial_{\tau} \phi \quad \text { on } \Gamma_{\text {slip }} \\
\phi_{t}+u_{\tau} \partial_{\tau} \phi=-V_{s} L(\phi) \quad \text { on } \Gamma_{\text {slip }} \\
u_{n}=0, \quad \partial_{n} \mu=0 \quad \text { on } \Gamma_{\text {slip }}
\end{gathered}
$$

where $L(\phi)=\varepsilon \partial_{n} \phi-\frac{\sqrt{2}}{6} \pi \cos \theta_{s} \cos \left(\frac{\pi}{2} \phi\right)$. In the above equations we have essentially six dimensionless parameters. These are $L_{d}=3 M \gamma / 2 \sqrt{2} V L_{0}^{2}, R=\rho V L_{0} / \eta, \lambda=3 \gamma / 2 \sqrt{2} \eta V$, which is inversely proportional to the capillary number $C_{a}=\eta V / \gamma, V_{s}=K \Gamma / V$, which is the ratio of $K \Gamma$ to $V, L_{s}=\eta / \beta L_{0}$, which is the ratio of slip length $l_{s}=\eta / \beta(\phi)$ to $L_{0}$ and $\varepsilon$.

Following [18], a second order finite element method is used to solve the coupled system numerically. For the sake of completeness, the main feature of the numerical scheme is 
introduced. In this work, the coupled Cahn-Hilliard and Navier-Stokes system is solved in a decoupled way. That is, for the Cahn-Hilliard equations, a second order semi-implicit temporal discretization with a convex splitting scheme is employed. In this scheme, a stabilizing parameter $s$ is introduced [17], which in the paper, has been given a value of 2.5. On the other hand, for the Navier-Stokes equations, a linearized semi-implicit scheme is used to avoid the non-linear iterations.

The second order of the temporal scheme for the system is formulated as follows.

$$
\begin{gathered}
\mu^{n+1}=-\varepsilon \Delta \phi^{n+1}+\frac{s}{\varepsilon} \phi^{n+1}-\frac{2(1+s)}{\varepsilon} \phi^{n}+\frac{(1+s)}{\varepsilon} \phi^{n-1}+\frac{2\left(\phi^{n}\right)^{3}}{\varepsilon}-\frac{\left(\phi^{n-1}\right)^{3}}{\varepsilon} \\
\frac{3 \phi^{n+1}-4 \phi^{n}+\phi^{n-1}}{2 \delta t}+2 \mathbf{u}^{n} \cdot \nabla \phi^{n+1}-\mathbf{u}^{n-1} \cdot \nabla \phi^{n}=L_{d} \Delta \mu^{n+1} \\
R\left(\frac{3 \mathbf{u}^{n+1}-4 \mathbf{u}^{n}+\mathbf{u}^{n-1}}{2 \delta t}+2 \mathbf{u}^{n} \cdot \nabla \mathbf{u}^{n+1}-\mathbf{u}^{n-1} \cdot \nabla \mathbf{u}^{n}\right)-\Delta \mathbf{u}^{n+1}+\nabla p^{n+1}=\lambda \mu^{n+1} \nabla \phi^{n+1} \\
\nabla \cdot \mathbf{u}^{n+1}=0
\end{gathered}
$$

And on the slip boundary,

$$
\begin{gathered}
\frac{3 \phi^{n+1}-4 \phi^{n}+\phi^{n-1}}{2 \delta t}+2 u_{\tau}^{n} \partial_{\tau} \phi^{n}-u_{\tau}^{n-1} \partial_{\tau} \phi^{n-1}=-V_{s} L\left(\phi^{n}\right) \\
\frac{\left(u_{\tau}^{s l i p}\right)^{n+1}}{L_{s}}=\lambda L\left(\phi^{n+1}\right) \partial_{\tau} \phi^{n+1}-\left(\partial_{n} u_{\tau}+\partial_{\tau} u_{n}\right)^{n+1} \\
u_{n}^{n+1}=0, \quad \partial_{n} \mu^{n+1}=0
\end{gathered}
$$

Here, $L\left(\phi^{n+1}\right)=\varepsilon \partial_{n} \phi^{n+1}-2 \times \frac{\sqrt{2}}{6} \pi \cos \theta_{s} \cos \left(\frac{\pi}{2} \phi^{n+1}\right)+\frac{\sqrt{2}}{6} \pi \cos \theta_{s} \cos \left(\frac{\pi}{2} \phi^{n}\right)$.

Mixed finite element (MFE) has been used in this work because of its favorable local conservation features. MFE allows the use of different polynomial degrees for the different variables in the governing equations (namely $\mathbf{u}, p$, and $\phi$ ). In this work a P2-P0 mixed finite elements space pair has been used to discretize the Navier-Stokes equations and P1-P1 space pair is used to discretize the Cahn-Hilliard equations. It has been shown by [19] that both space pairs are stable because they satisfy the inf-sup stable condition. Furthermore, the P1-P1 space pair used for the Cahn-Hilliard equation also fulfills the following conservation law for the computational domain without inflow and outflow [20]. 


$$
\int_{\Omega} \phi_{h}^{m} d \mathbf{x} \equiv \int_{\Omega} \phi_{h}^{0} d \mathbf{x} \quad \text { for } m \geq 1
$$

On the other hand, for the Naiver-Stokes equations, with the P2-P0 space pair, the accuracy of the velocity is second order and the pressure approximation is of first order.

MFE formulation requires defining the following spaces to discretize the coupled system spatially, these are

$$
\begin{gathered}
M_{h}=\left\{q_{h} \in L_{0}^{2}(\Omega) ;\left.q_{h}\right|_{K} \in P_{0}(K)\right\} \\
U_{h}=\left\{v_{h} \in C^{0}(\bar{\Omega}) ;\left.v_{h}\right|_{K} \in P_{2}(K)\right\} \\
W_{h}=\left\{w_{h} \in C^{0}(\bar{\Omega}) ;\left.w_{h}\right|_{K} \in P_{1}(K)\right\}
\end{gathered}
$$

where $\Omega$ is the computational domain and $L_{0}^{2}(\Omega)=\left\{v \in L^{2}(\Omega) ; \int_{\Omega} v d \mathbf{x}=0\right\}$.

The finite element discretization for the Navier-Stokes equations with the GNBC is defined as follows, find $\left(\mathbf{u}_{h}, p_{h}\right) \in\left(U_{h}\right)^{2} \times M_{h}$, where $\mathbf{u}_{h}=\left(u_{x}, u_{y}\right)$, such that, for $\forall\left(\mathbf{v}_{h}, q_{h}\right) \in\left(U_{h}\right)^{2} \times M_{h}$

$$
\begin{aligned}
& R\left(\frac{3 \mathbf{u}_{h}^{n+1}-4 \mathbf{u}_{h}^{n}+\mathbf{u}_{h}^{n-1}}{2 \delta t}, \mathbf{v}_{h}\right)+2 R\left(\mathbf{u}_{h}^{n} \cdot \nabla \mathbf{u}_{h}^{n+1}, \mathbf{v}_{h}\right)-R\left(\mathbf{u}_{h}^{n-1} \cdot \nabla \mathbf{u}_{h}^{n}, \mathbf{v}_{h}\right)+\left(\nabla \mathbf{u}_{h}^{n+1}, \nabla \mathbf{v}_{h}\right)-\left(p_{h}^{n+1}, \nabla \cdot \mathbf{v}_{h}\right) \\
& +\left\langle\frac{1}{L_{s}} u_{\tau}^{n+1}, v_{h}\right\rangle_{\Gamma \text { slip }}=\lambda\left(\mu_{h}^{n+1} \nabla \phi_{h}^{n+1}, \mathbf{v}_{h}\right)+\left\langle\left(\lambda L\left(\phi_{h}^{n+1}\right) \partial_{\tau} \phi_{h}^{n+1}, v_{h}\right\rangle_{\Gamma_{\text {slip }}}-\left(\left\langle P_{2}, v_{h}\right\rangle_{\Gamma_{\text {oultet }}}-\left\langle P_{1}, v_{h}\right\rangle_{\Gamma_{\text {inlet }}}\right)\right.
\end{aligned}
$$

$$
\left(\nabla \cdot \mathbf{u}_{h}^{n+1}, q_{h}\right)=0
$$

Likewise, the weak formulation for the Cahn-Hilliard equations with the GNBC boundary condition is defined as follows, find $\left(\phi_{h}, \mu_{h}\right) \in W_{h} \times W_{h}$, such that, for $\forall w_{h} \in W_{h}$

$$
\begin{gathered}
\left(\frac{3 \phi_{h}^{n+1}-4 \phi_{h}^{n}+\phi_{h}^{n-1}}{2 \delta t}, w_{h}\right)+\left(2 \mathbf{u}_{h}^{n} \cdot \nabla \phi_{h}^{n+1}, w_{h}\right)-\left(\mathbf{u}_{h}^{n-1} \cdot \nabla \phi_{h}^{n}, w_{h}\right)=-L_{d}\left(\nabla \mu_{h}^{n+1}, \nabla w_{h}\right) \\
\left(\mu_{h}^{n+1}, w_{h}\right)=\varepsilon\left(\nabla \phi_{h}^{n+1}, \nabla w_{h}\right)+\frac{s}{\varepsilon}\left(\phi_{h}^{n+1}, w_{h}\right)+\left(-\frac{2(1+s)}{\varepsilon} \phi_{h}^{n}+\frac{(1+s)}{\varepsilon} \phi_{h}^{n-1}+\frac{2\left(\phi_{h}^{n}\right)^{3}}{\varepsilon}-\frac{\left(\phi_{h}^{n-1}\right)^{3}}{\varepsilon}, w_{h}\right) \\
+\frac{1}{V_{s}}\left[\left\langle\frac{3 \phi_{h}^{n+1}-4 \phi_{h}^{n}+\phi_{h}^{n-1}}{2 \delta t}, w_{h}\right\rangle_{\Gamma_{s l i p}}+\left\langle 2 u_{\tau}^{n} \partial_{\tau} \phi_{h}^{n}-u_{\tau}^{n-1} \partial_{\tau} \phi_{h}^{n-1}, w_{h}\right\rangle_{\Gamma_{s l i p}}\right] \\
+\left\langle-2 \times \frac{\sqrt{2}}{6} \pi \cos \theta_{s} \cos \left(\frac{\pi}{2} \phi_{h}^{n}\right)+\frac{\sqrt{2}}{6} \pi \cos \theta_{s} \cos \left(\frac{\pi}{2} \phi_{h}^{n-1}\right), w_{h}\right\rangle_{\Gamma_{\text {slip }}}
\end{gathered}
$$

The discretized weak forms generate two linear systems for the Cahn-Hilliard and the Navier- 
Stokes equations, respectively. The direct linear solver package, UMFPACK [27], which is an efficient solver based on the sparse LU factorization, is used to solve the linear systems. It is to be noted that the time step has been chosen such that the CFL condition is generally respected. More discussion on the accuracy of the numerical methods can be found in [14, 17 and 18].

\section{Results and discussion}

The geometrical domain considered in this work represents a horizontal channel of unit length subject to fixed pressure boundary conditions at both the inlet and the exit sections. The channel is initially filled with a non-wetting fluid (blue) with a precursor wetting layer at the walls (in red) as shown in Fig. 2. A wetting fluid (of the same type as that of the precursor layer) is allowed to enter the channel from the left. Three basic scenarios are considered in this work. The first scenario is devoted to studying the effect of wettability condition of the wall to the red phase without the precursor layer. The second scenario, on the other hand, is devoted to studying the effect of wettability of the channel walls of the flow of the red phase when there exist a precursor layer of the red fluid. The last scenario is to investigate the effect of the precursor layer thickness on the flow of this two phase system. In these systems the moving contact line plays crucial rule. The precursor layer, results in the contact line to break (disappear) as the red phase merges with the wetting precursor layer as shown schematically in Fig. 3.

\subsection{Effects of wettability conditions without the precursor layer.}

In this case the walls of the channel have been assigned several wettability conditions with contact angels ranging from $10^{\circ}$ to $80^{\circ}$ for wetting scenarios and from $100^{\circ}$ to $160^{\circ}$ for the nonwetting scenarios. In all these cases the system operates under the same fixed static pressure at the inlet and at the exit. Figure 4 shows snap shots of the configuration of the two phase system including the interface for the different wettability conditions. It is clear that the interface assumes complex configurations depending on the wettability conditions. One can notice that the position of contact line along the channel walls, when the wettability is large, is approximately at the same distance from the origin as that of the interface at the center of the channel and gradually recedes when the wettability becomes less. For the non-wetting scenarios, the contact line retards clearly than it is at the center. This behavior of the contact line has interesting implication on the flow characteristics of this two phase system. Clearly it seems that, at the 
entrance region, the flow accelerates faster when the wettability is large. This behavior is manifested in Fig. 5 which shows the threshold of the red phase arrival at the exit. In this figure, it is clear that the red phase reaches the exit earlier when the wettability is larger.

\subsection{Effects of wettability conditions with the precursor layer.}

In these scenarios, a thin layer of the red phase is initially wetting the channel walls. The precursor layer is assumed of a thickness of 0.1 length units. Different wettability conditions have been tested with contact angles ranging from $10^{\circ}$ up to $80^{\circ}$. It is worth mentioning that a non-wetting precursor layer will not be stable and will not stay attached to the wall and therefore for all the precursor layer scenarios, wetting conditions have been studied. For these scenarios, when the flow starts, the red phase at the inlet moves faster than the precursor layer and eventually merges with it. Upon merging, the contact line disappears and the flow of the red phase is facilitated even more. In other words, the precursor layer is working as a lubricating fluid to facilitate the movement of the red phase. On the other hand, the moving contact line at the far edge of the precursor layer is moving faster the larger the wettability (i.e., the smaller the contact angle). This allows the red phase to advance more when the wettability is large as depicted in Fig. 6. It is noteworthy that, for larger contact angle wetting scenarios, the precursor layer tries to converge because it does not have large affinity to the solid walls.

Breakthrough curves of the red phase are shown in Fig. 7 below. It is clear that the red phase reaches the exit of the channel faster when the wettability is larger. On comparing the same wettability scenarios with and without the precursor layer, one can clearly see that the red phase is even faster with the precursor layer than it is without the layer, Fig. 8. This can also be seen in Fig. 9 which shows the breakthrough time with and without the precursor layer and it, again, confirms that the precursor layer facilitates the motion of the red phase more than it is without such layer.

\subsection{Effects of the thickness of the precursor layer.}

In these scenarios, the effects of the precursor layer thickness are investigated. The thickness of the precursor layer spans a range from 0.06 up to 0.16 length units and the contact angle for all these scenarios have been considered $60^{\circ}$. When the thickness of the precursor layer is small, the movement of this layer is restricted as the contact line at the wall interface is not advancing fast. 
For larger precursor layer thickness the movement of the contact line becomes more faster as depicted in Fig. 10. Furthermore, as the red phase at the inlet advances until eventually merging with the precursor layer, the contact line disappears. Apparently the motion of the red phase at the core of the channel is also advancing faster the larger the thickness of the precursor layer which can also be seen in Fig. 10.

Figure 11 shows breakthrough curves of the red phase at the exit of the channel for the different thicknesses of the precursor layer. Again, it is clear that the thickness of the precursor layer has appreciable effect on the breakthrough time of the red phase which reaches the exit faster the larger the thickness of the precursor layer.

\section{Conclusions}

The problem of two phase flow of two immiscible fluids in a horizontal channel is investigated numerically with respect to the wettability condition at the solid wall and with respect to the effects of a precursor wetting fluid layer. Interesting results are obtained that can be summarized as

1. The wettability condition at the wall very much influences the configuration of the interface, the acceleration of the two phase system at the entrance region and the breakthrough time of the phases.

2. When the wettability is large the contact line moves faster than it is when the wettability is small.

3. The existence of a precursor wetting layer very much facilitate the motion of the phases.

4. The thickness of the precursor layer influences the breakthrough time of the phases. 


\section{References}

[1] A. Salama and P. J. Van Geel, Flow and Solute Transport in Saturated Porous Media: 1. The Continuum Hypothesis, J. Porous Media, 11, 4, 403-413, 2008

[2] A. Salama and P. J. Van Geel, Flow and Solute Transport in Saturated Porous Media: 2. Violating the Continuum Hypothesis, J. Porous Media, 11, 5, 421-441, 2008.

[3] M.F. El-Amin, A. Salama, and S. Sun, Solute Transport with Chemical Reaction in Single and Multi-Phase Flow in Porous Media, in Mass Transfer in Multiphase Systems and its Applications, ed. M.F. El Amin, 2011.

[4] C. Huh and S.G. Mason, The steady movement of a liquid meniscus in a capillary tube. J. Fluid Mech., 81:401-419, 1977.

[5] E.B. Dussan, V., On the spreading of liquids on solid surfaces: static and dynamic contact lines. Ann. Rev. Fluid Mech., 11:371-400, 1979.

[6] J.F. Joanny and P.G.de Gennes, A model for contact angle hysteresis. J. Chem. Phys., 81:552-562, 1984.

[7] R.G. Cox, The dynamics of the spreading of liquids on a solid surface. Part 1. Viscous flow. J. Fluid. Mech., 168:169-194, 1986.

[8] J. Koplik, J.R. Banavar, and J.F. Willemsen, Molecular dynamics of poiseuille flow and moving contact lines. Phys. Rev. Lett., 60:1282-1285, 1988.

[9] P.A. Thompson and M.O. Robbins, Simulations of contact-line motion: slip and the dynamic contact angle. Phys. Rev. Lett., 63:766-769, 1989.

[10] P.A. Thompson, W.B. Brinckerhoff, and M.O. Robbins, Microscopic studies of static and dynamic contact angles. J. Adhesion Sci. Tech., 7:535-554, 1993.

[11] H.Y. Chen, D. Jasnow, and J. Vinals, Interface and contact line motion in a two phase fluid under shear flow. Phys. Rev. Lett., 85:1686-1689, 2000.

[12] D. Jacqmin, Contact-line dynamics of a diffuse fluid interface. J. Fluid. Mech., 402:57-88, 2000.

[13] Q. He and X.-Ping Wang, Numerical study of the effect of Navier slip on the driven cavity flow; Z. Angew, Math. Mech, 89, 10, pp. 857 - 868, 2009.

[14] T. Qian, X. P. Wang, and P. Sheng, "Molecular scale contact line hydrodynamics of immiscible flows," Phys. Rev. E 68, 016306, 2003. 
[15] T. Qian, C. Wu, S. L. Lei, X.-P. Wang, and P. Sheng, Modeling and simulations for molecular scale hydrodynamics of moving contact line in immiscible two phase flows, Journal of Physics: Condensed Matter 21, 464119 (2009).

[16] H. Mehrabian, P. Gao, and J. J. Feng, Wicking flow through microchannels, Physics of Fluids 23, 122108, 2011.

[17] M. Gao, X.-P. Wang, “A Gradient Stable Scheme for a Phase Field Model for the Moving Contact Line Problem”, J. Computational Physics, 231, 4, pp. 1372-1386, 2012.

[18] K. Bao, Y. Shi, S. Sun, X.-P. Wang, A finite element method for the numerical solution of the coupled cahn-hilliard and Navier-Stokes system for moving contact line problems, J. Computational Physics, 231, 24, pp. 8083-8099, 2012.

[19] F. Xiaobing and A. Prohl, "Error Analysis of a Mixed Finite Element Method for the CahnHilliard Equations". Numer. Math, 99, 1, pp. 47-84, 2004

[20] F. Xiaobing, Fully discrete finite element approximations of the Navier-Stokes-CahnHilliard diffuse interface model for two phase flows. SIAM J. Numer Anal, 44, 3, pp. 1049-1072, 2006

[21] P. A. Thompson and M. O. Robbins, Simulations of contact-line motion: slip and the dynamic contact angle, Phys. Rev. Lett., 63, (1989)

[22] P. A. Thompson and M. O. Robbins, Shear flow near solids: epitaxial order and flow boundary conditions, Phys. Rev. A, 41, p. 6830, (1990).

[23] J-L. Barrat and L. Bocquet, Large slip effect at a nonwetting fluid-solid interface, Phys. Rev. Lett., 82, p. 4671, (1999)

[24] M. Cieplak, J. Koplik and J. R. Banavar, Boundary conditions at a fluid-solid interface, Phys. Rev. Lett., 86, p. 803, (2001).

[25] T. Qian, X.-P., Wang, P. Sheng, Molecular hydrodynamics of the moving contact line in two-phase immiscible flows, Comm. In Computational Physics, 1, 1, p. 1-52, (2006)

[26] T. Qian, X. P. Wang, and P. Sheng, "Molecular scale contact line hydrodynamics of immiscible flows," Phys. Rev. E 68, 016306, 2003.

[27] T. A. Davis, A column pre-ordering strategy for the unsymmetric-pattern multifrontal method, ACM Transactions on Mathematical Software, 30, 2, pp. 165-19, 2004. 


\section{List of Figures}

Fig. 1 Schematic of the fluid slip near the contact line. A solid wall is moving to the left at velocity $\mathrm{U}$. The arrows show the relative magnitude of the velocity of the fluid and the solid wall (not to scale).

Fig. 2 Schematic of the computational domain. The layer of red fluid phase at the solid surface is of the same fluid type entering from the left.

Fig. 3 Disappearance of the contact line when the incoming phase merges with the precursor wetting layer.

Fig. 4 Effect of wettability conditions with no precursor layer. The interface assumes complex configuration that is very much influenced by the wettability condition at the interface.

Fig. 5 Breakthrough curves of the red phase for different wettability conditions with no precursor layer.

Fig. 6 Phase diagram evolution for different contact angle with the precursor layer.

Fig. 7 Breakthrough curves of the red phase for different wettability conditions with the precursor layer.

Fig. 8 Breakthrough curves of the red phase for different wettability conditions with and without the precursor layer.

Fig. 9 Comparisons of the time at breakthrough of the red phase for different wettability conditions with and without the precursor layer.

Fig.10 Phase diagram evolution for different thickness of the precursor layer.

Fig. 11 Breakthrough curves for red phase for different thickness of the precursor wetting layer. 


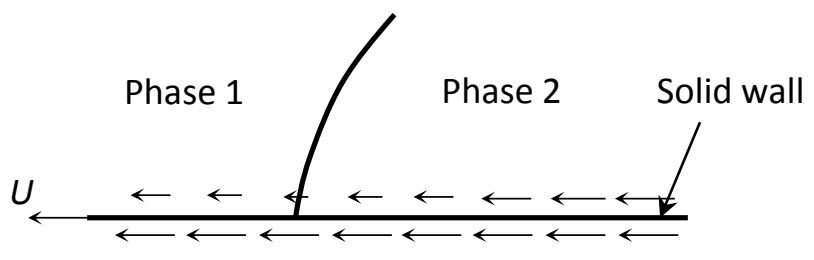

Fig. 1 Schematic of the fluid slip near the contact line. A solid wall is moving to the left at velocity $U$. The arrows show the relative magnitude of the velocity of the fluid and the solid wall (not to scale).

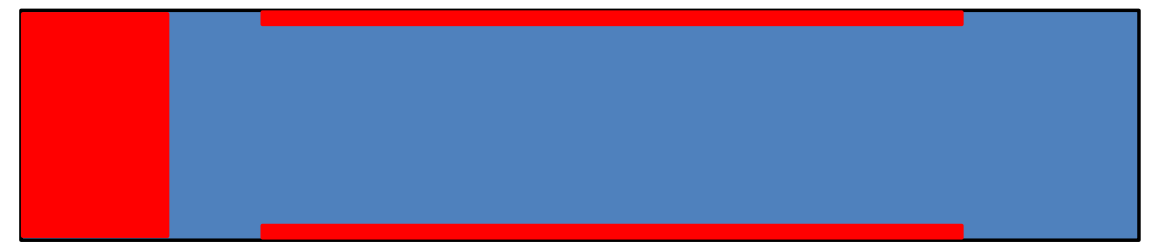

Fig. 2 Schematic of the computational domain. The layer of red fluid phase at the solid surface is of the same fluid type entering from the left.

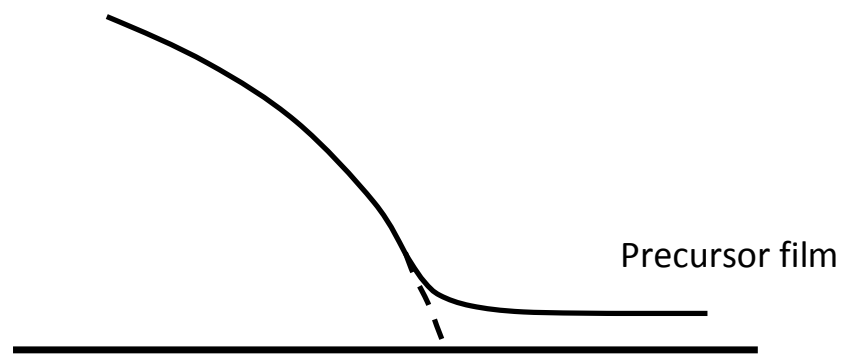

Fig. 3 Disappearance of the contact line when the incoming phase merges with the precursor wetting layer 


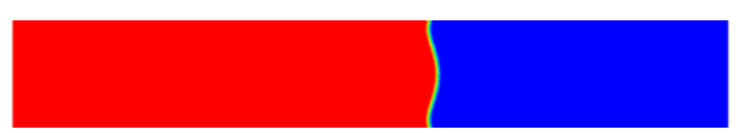

Contact angle 20

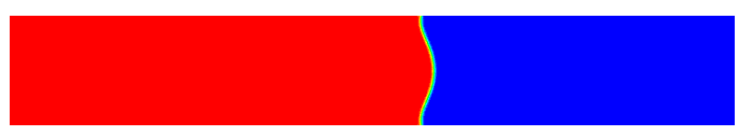

Contact angle 40

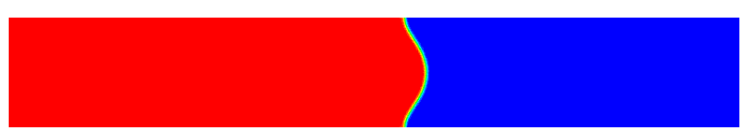

Contact angle 60

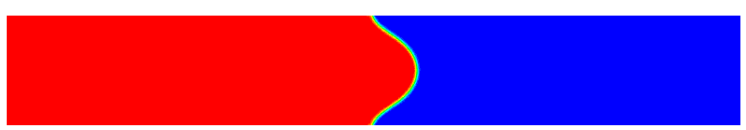

Contact angle 80

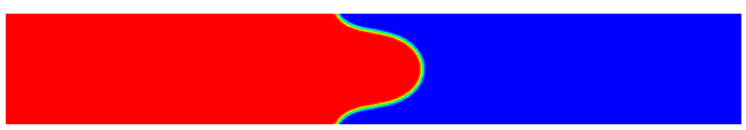

Contact angle 90

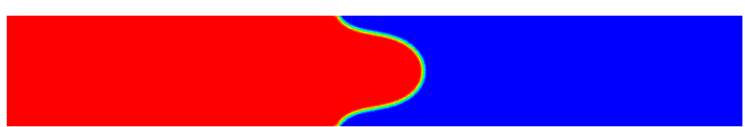

Contact angle 90

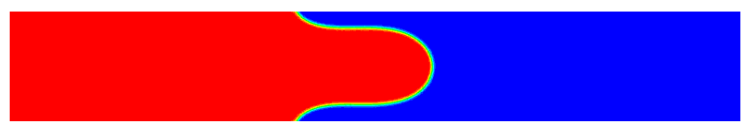

Contact angle 100

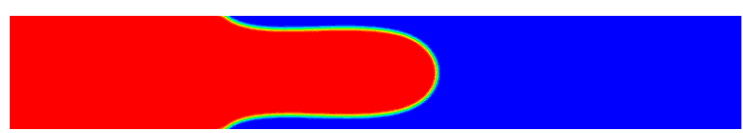

Contact angle 120

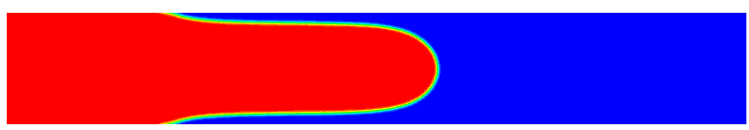

Contact angle 140

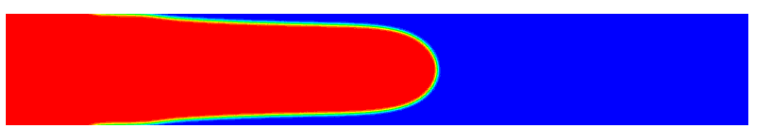

Contact angle 160

Fig. 4 Effect of wettability conditions with no precursor layer. The interface assumes complex configuration that is very much influenced by the wettability condition at the interface. 


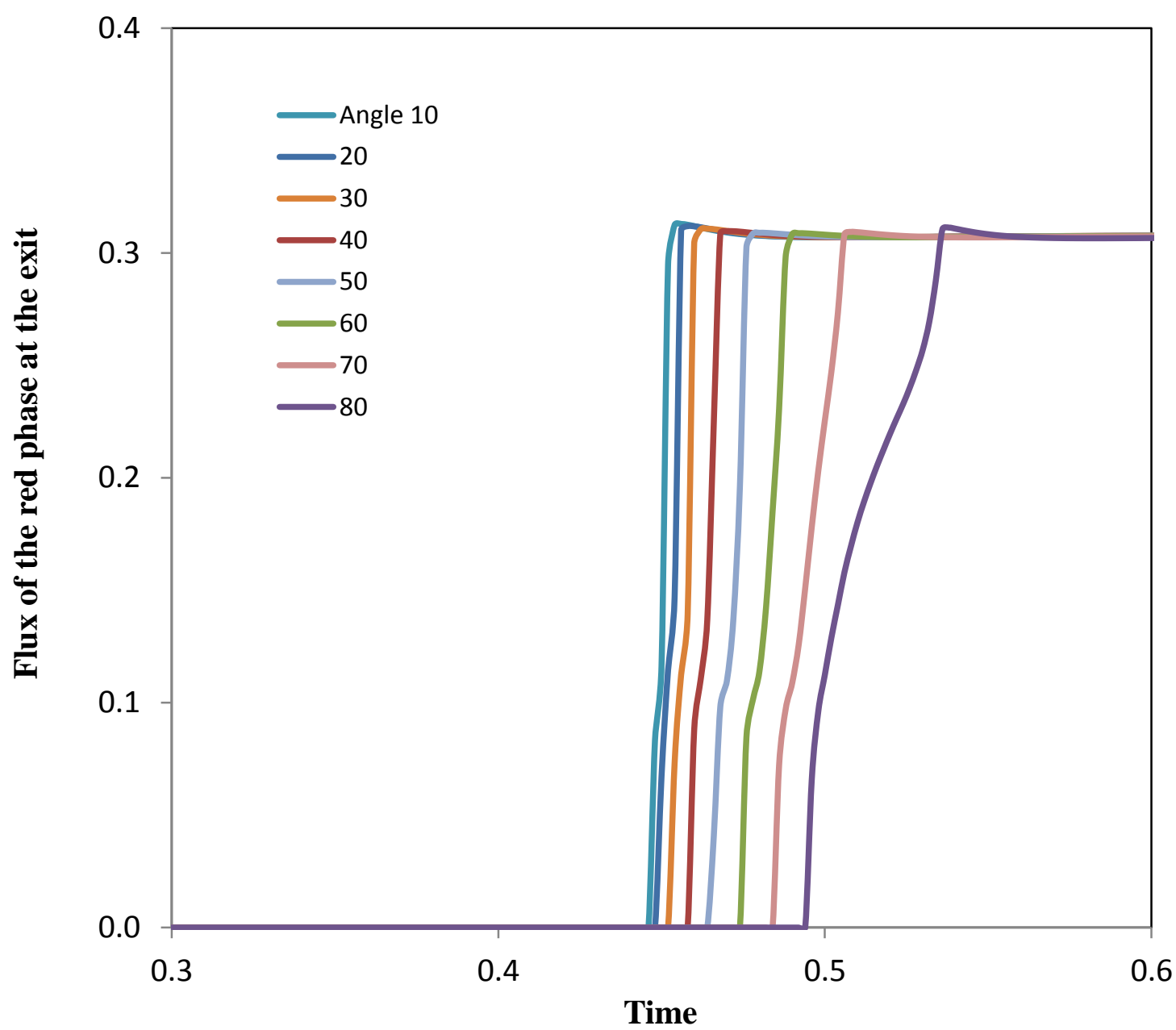

Fig. 5 Breakthrough curves of the red phase for different wettability conditions with no precursor layer. 

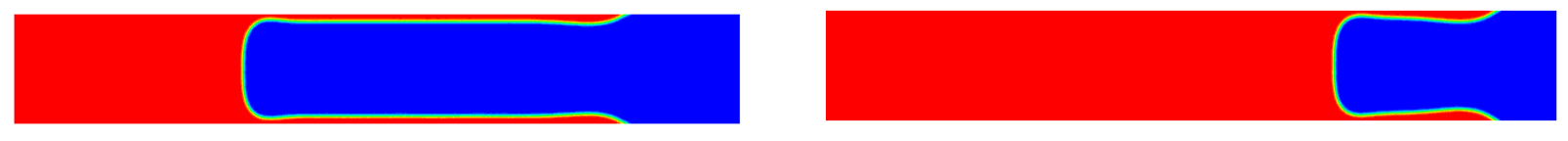

Contact angle 20
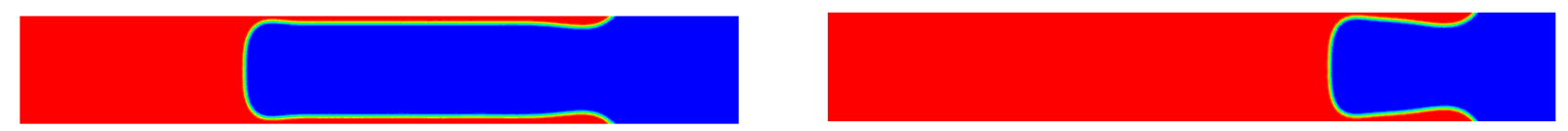

Contact angle 40
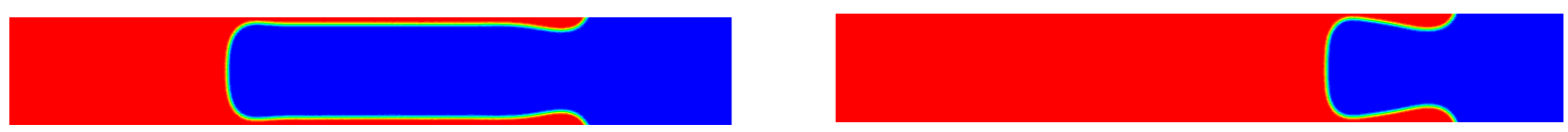

Contact angle 60
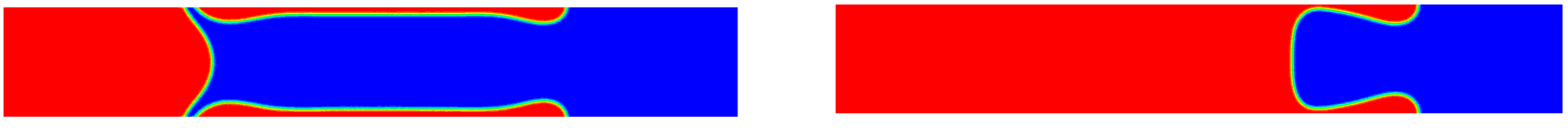

Contact angle 80
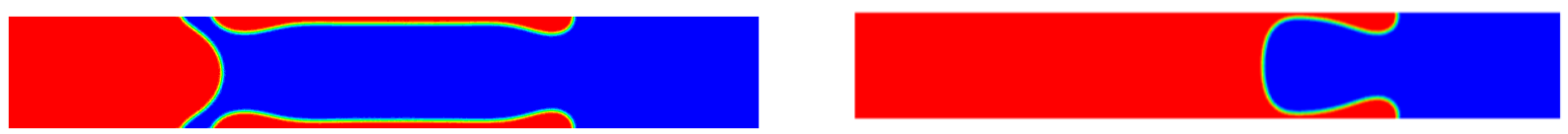

Contact angle 90

After 10 units of time

After 25 units of time

Fig.6 Phase diagram evolution for different contact angle with the precursor layer. 


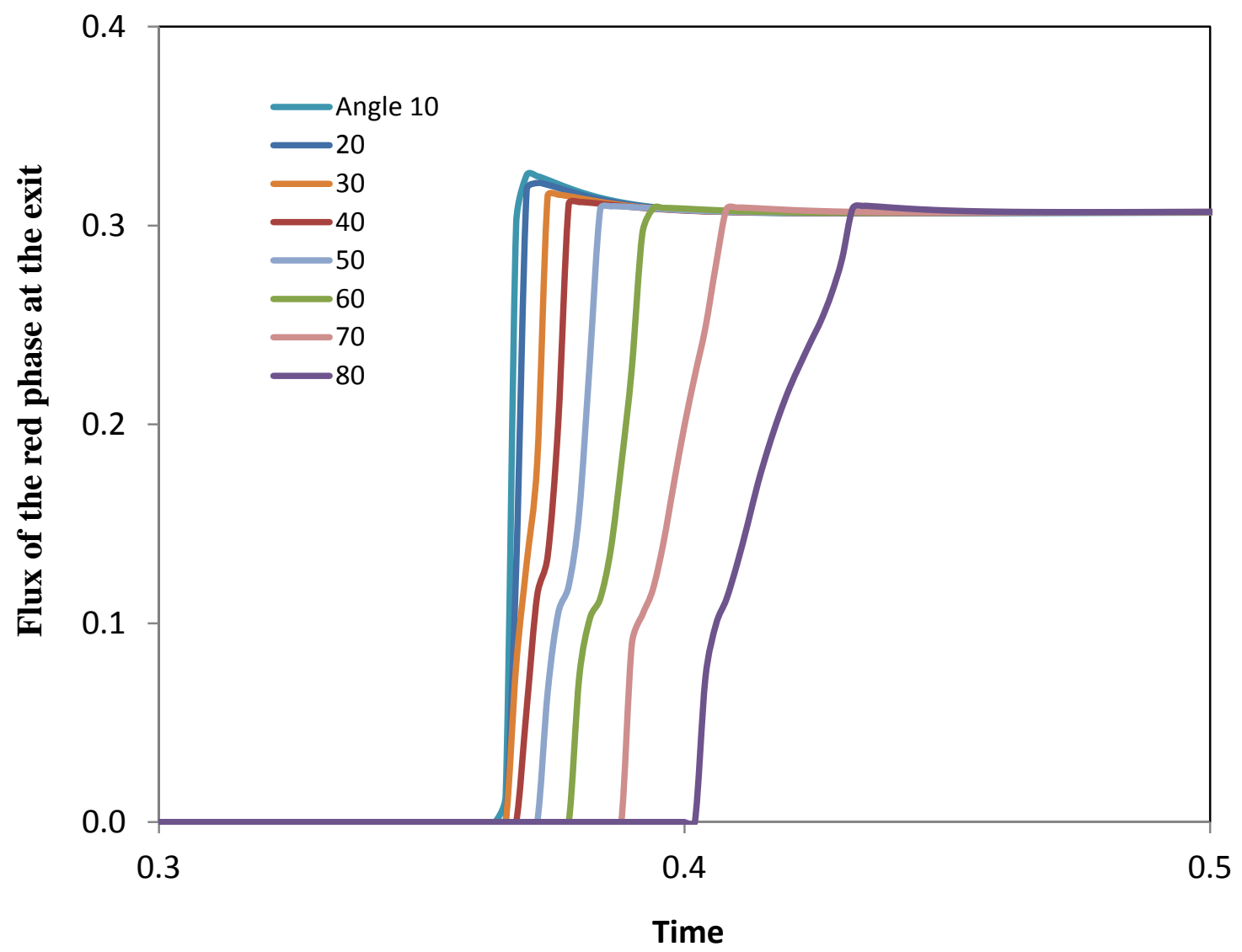

Fig. 7 Breakthrough curves of the red phase for different wettability conditions with the precursor layer. 


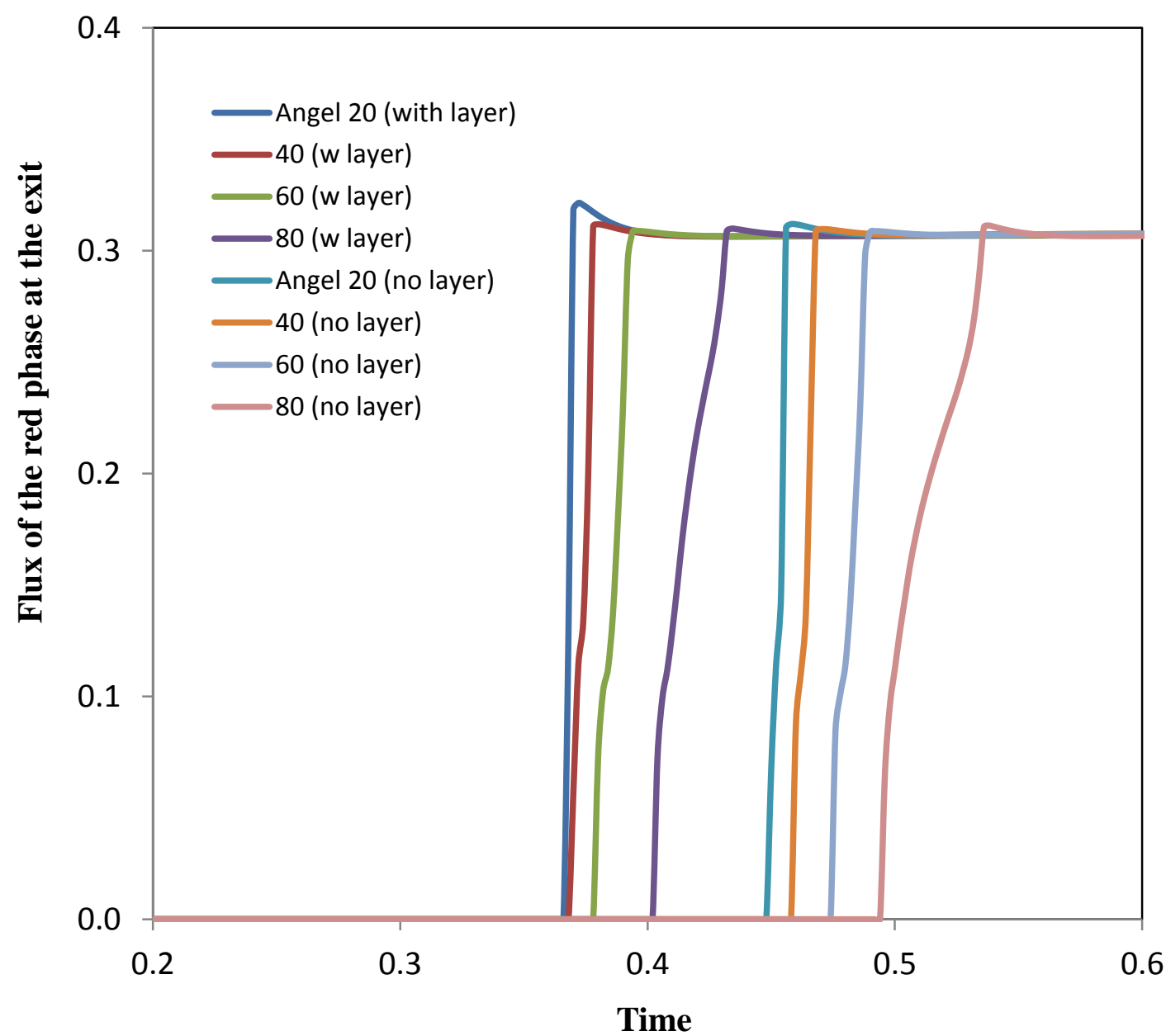

Fig. 8 Breakthrough curves of the red phase for different wettability conditions with and without the precursor layer. 


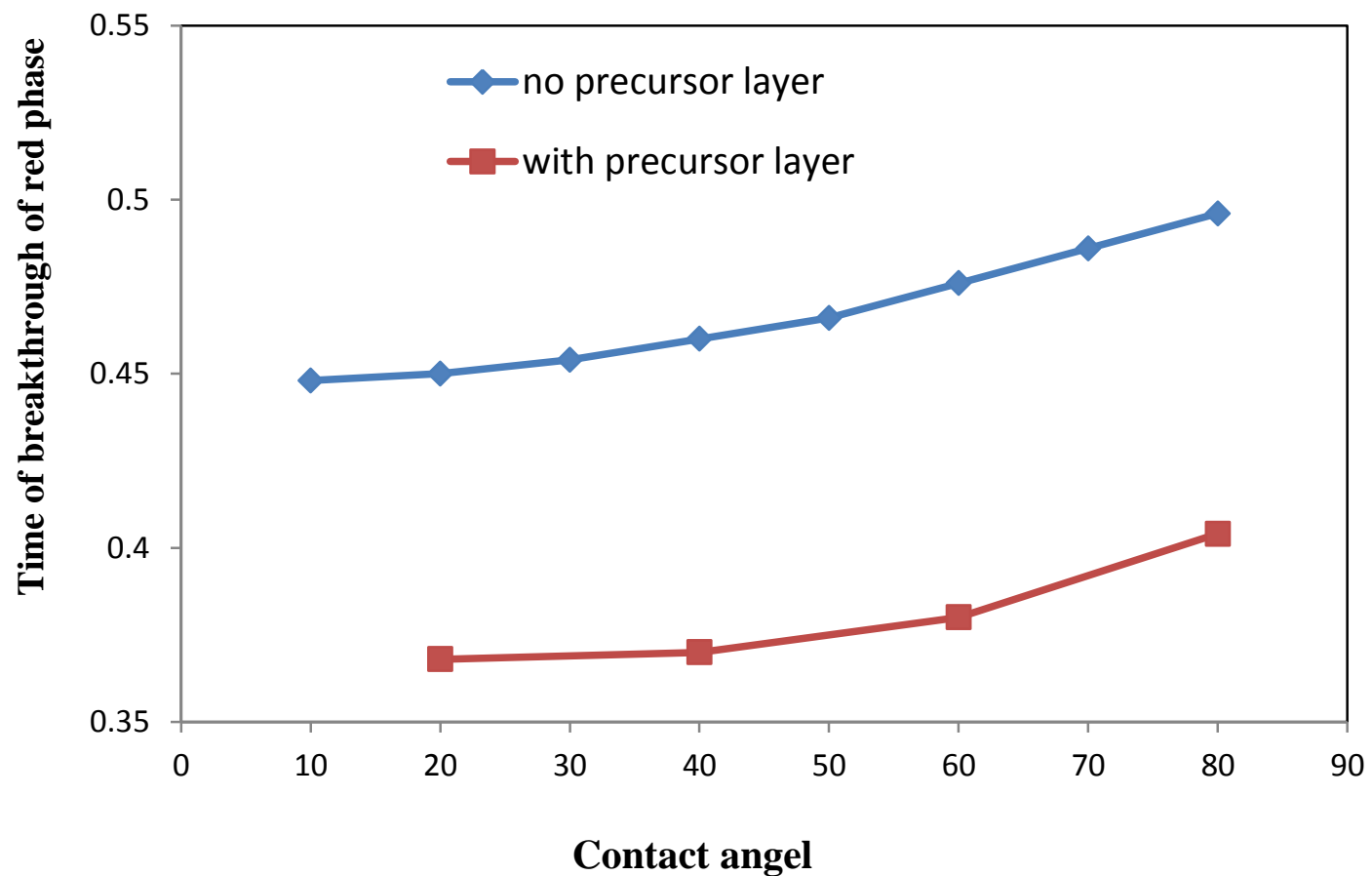

Fig. 9 Comparisons of the time at breakthrough of the red phase for different wettability conditions with and without the precursor layer. 

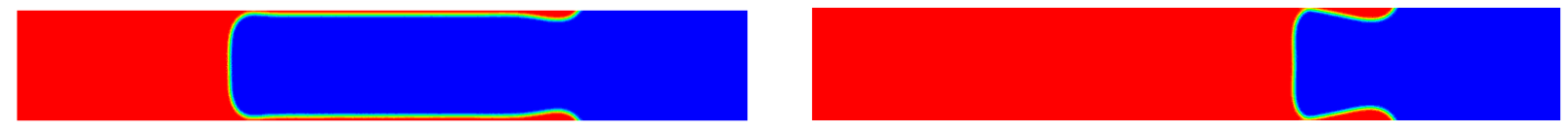

Thickness 0.06

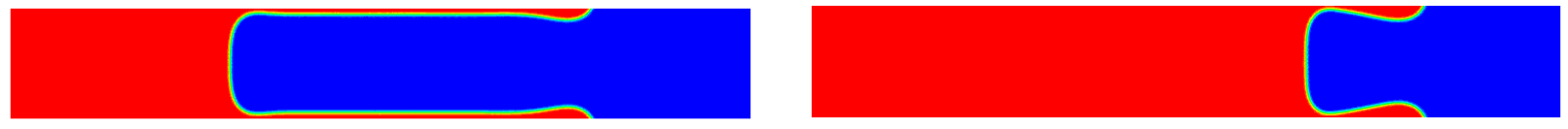

Thickness 0.08
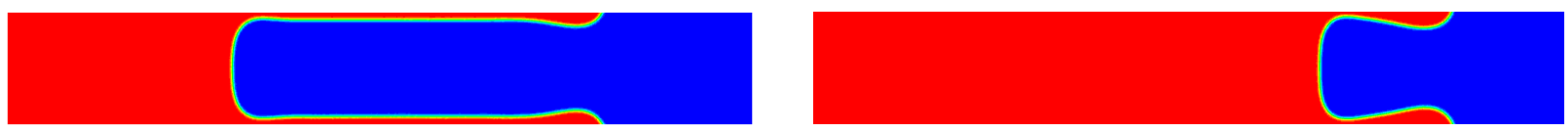

Thickness 0.1
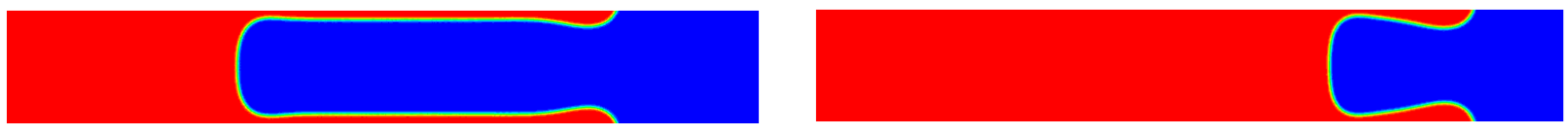

Thickness 0.12
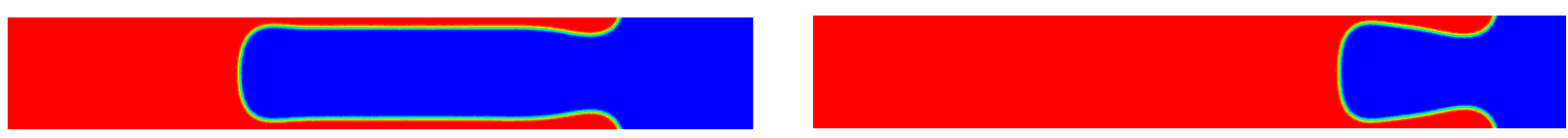

Thickness 0.14
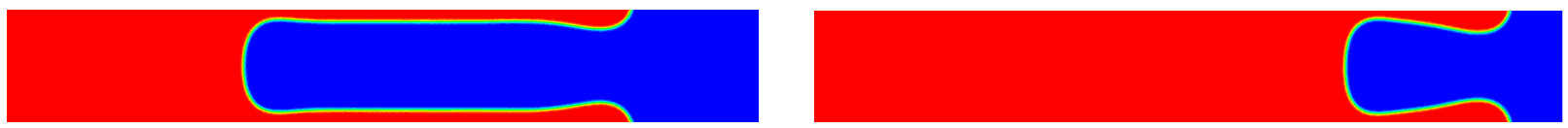

Thickness 0.16

After 10 units of time

After 25 units of time

Fig.10 Phase diagram evolution for different thickness of the precursor layer 


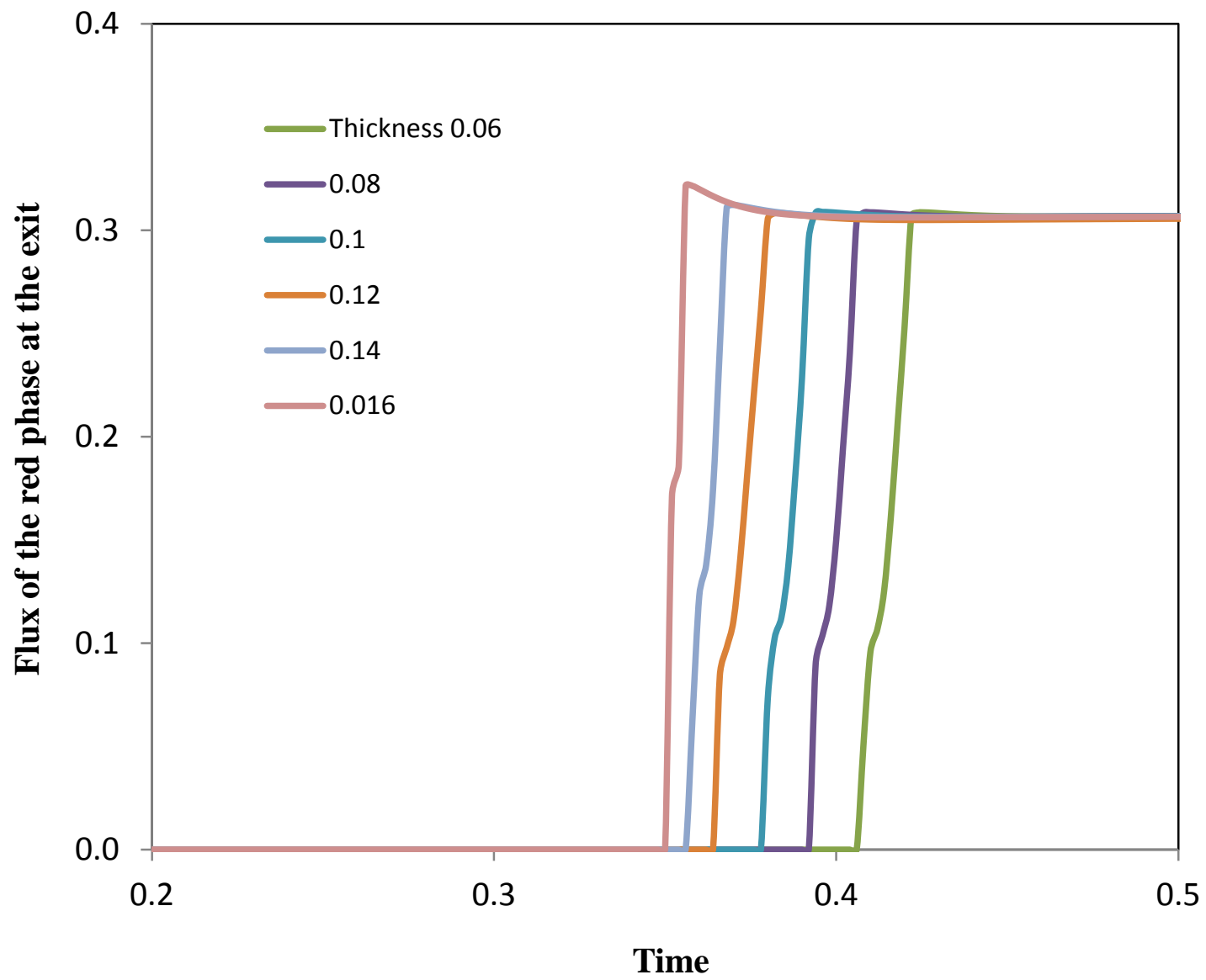

Fig. 11 Breakthrough curves for red phase for different thickness of the precursor wetting layer. 